\title{
Multi-Eulerian tours of directed graphs
}

\author{
Matthew Farrell Lionel Levine* \\ Department of Mathematics \\ Cornell University \\ Ithaca, New York, USA \\ msf235@cornell. edu \\ http://www. math. cornell.edu/ levine
}

Submitted: Sep 24, 2015; Accepted: Apr 14, 2016; Published: Apr 29, 2016

Mathematics Subject Classifications: 05C05, 05C20, 05C30, 05C45, 05C50

\begin{abstract}
Not every graph has an Eulerian tour. But every finite, strongly connected graph has a multi-Eulerian tour, which we define as a closed path that uses each directed edge at least once, and uses edges $e$ and $f$ the same number of times whenever tail $(e)=\operatorname{tail}(f)$. This definition leads to a simple generalization of the BEST Theorem. We then show that the minimal length of a multi-Eulerian tour is bounded in terms of the Pham index, a measure of 'Eulerianness'.
\end{abstract}

Keywords: BEST theorem, coEulerian digraph, Eulerian digraph, Eulerian path, Laplacian, Markov chain tree theorem, matrix-tree theorem, oriented spanning tree, period vector, Pham index, rotor walk

In the following $G=(V, E)$ denotes a finite directed graph, with loops and multiple edges permitted. An Eulerian tour of $G$ is a closed path that traverses each directed edge exactly once. Such a tour exists only if the indegree of each vertex equals its outdegree; the graphs with this property are called Eulerian. The BEST theorem (named for its discoverers: de Bruijn, Ehrenfest [4], Smith and Tutte [12]) counts the number of such tours. The purpose of this note is to generalize the notion of Eulerian tour and the BEST theorem to any finite, strongly connected graph $G$.

Definition 1. Fix a vector $\pi \in \mathbb{N}^{V}$ with all entries strictly positive. A $\boldsymbol{\pi}$-Eulerian tour of $G$ is a closed path that uses each directed edge $e$ of $G$ exactly $\pi_{\text {tail }(e)}$ times.

Note that existence of a $\pi$-Eulerian tour implies that $G$ is strongly connected: for each $v, w \in V$ there are directed paths from $v$ to $w$ and from $w$ to $v$. We will show that,

${ }^{*}$ Supported by NSF grant DMS-1455272 and a Sloan Fellowship. 
conversely, every strongly connected graph $G$ has a $\pi$-Eulerian tour for suitable $\pi$. To do so, recall the BEST theorem counting 1-Eulerian tours of an Eulerian directed multigraph $G$. Write $\epsilon_{\pi}(G, e)$ for the number of $\pi$-Eulerian tours of $G$ starting with a fixed edge $e$.

Theorem 1. (BEST $[4,12])$ A strongly connected multigraph $G$ has a 1-Eulerian tour if and only if the indegree of each vertex equals its outdegree, in which case the number of such tours starting with a fixed edge e is

$$
\epsilon_{\mathbf{1}}(G, e)=\kappa_{w} \prod_{v \in V}\left(d_{v}-1\right) !
$$

where $d_{v}$ is the outdegree of $v$; vertex $w$ is the tail of edge e, and $\kappa_{w}$ is the number of spanning trees of $G$ oriented toward $w$.

A spanning tree oriented toward $\boldsymbol{w}$ is a set of edges $t$ such that $w$ has outdegree 0 in $t$, each vertex $v \neq w$ has outdegree 1 in $t$, and $t$ has no directed cycles. Let us remark that for a general directed graph the number $\kappa_{w}$ of spanning trees oriented toward $w$ depends on $w$, but for an Eulerian directed graph it does not (since $\epsilon_{\mathbf{1}}(G, e)$ does not depend on $e$ ).

The graph Laplacian is the $V \times V$ matrix

$$
\Delta_{u v}= \begin{cases}d_{v}-d_{v v}, & u=v \\ -d_{v u} & u \neq v\end{cases}
$$

where $d_{v u}$ is the number of edges directed from $v$ to $u$, and $d_{v}=\sum_{u} d_{v u}$ is the outdegree of $v$. By the matrix-tree theorem $[10,5.6 .8], \kappa_{w}$ is the determinant of the submatrix of $\Delta$ omitting row and column $w$.

Thus, the BEST and matrix-tree theorems give a computationally efficient exact count of the 1-Eulerian tours of a directed multigraph. (In contrast, exact counting of undirected Eulerian tours is a \#P-complete problem!)

Observing that the 'indegree=outdegree' condition in the BEST theorem is equivalent to $\Delta \mathbf{1}=\mathbf{0}$ where $\mathbf{1}$ is the all ones vector, we arrive at the statement of our main result.

Theorem 2. Let $G=(V, E)$ be a strongly connected directed multigraph with Laplacian $\Delta$, and let $\pi \in \mathbb{N}^{V}$. Then $G$ has a $\pi$-Eulerian tour if and only if

$$
\Delta \pi=\mathbf{0} .
$$

If $\Delta \pi=\mathbf{0}$, then the number of $\pi$-Eulerian tours starting with edge $e$ is given by

$$
\epsilon_{\pi}(G, e)=\kappa_{w} \prod_{v \in V} \frac{\left(d_{v} \pi_{v}-1\right) !}{\left(\pi_{v} !\right)^{d_{v}-1}\left(\pi_{v}-1\right) !}
$$

where $d_{v}$ is the outdegree of $v$; vertex $w$ is the tail of edge e, and $\kappa_{w}$ is the number of spanning trees of $G$ oriented toward $w$. 
Note that the ratio on the right side is a multinomial coefficient and hence an integer.

The proof below is a straightforward application of the BEST theorem. The same proof device of constructing an Eulerian multigraph from a strongly connected graph was used in [2, Theorem 3.18] to relate the Riemann-Roch property of 'row chip-firing' to that of 'column chip-firing'. In the remainder of the paper we find the length of the shortest $\pi$ Eulerian tour (Theorem 5) and conclude with two mild generalizations: $\lambda$-Eulerian tours (Theorem 6) and $\pi$-Eulerian paths (Theorem 7 ).

Proof of Theorem 2. Define a multigraph $\widetilde{G}$ by replacing each edge $e$ of $G$ from $u$ to $v$ by $\pi_{u}$ edges $e^{1}, \ldots, e^{\pi_{u}}$ from $u$ to $v$. Since $\pi$ has all positive entries, $\widetilde{G}$ is strongly connected. Each vertex $v$ of $\widetilde{G}$ has outdegree $d_{v} \pi_{v}$ and indegree $\sum_{u \in V} \pi_{u} d_{u v}$, so $\widetilde{G}$ is Eulerian if and only if $\Delta \pi=\mathbf{0}$.

If $\left(e_{1}^{i_{1}}, \ldots, e_{m}^{i_{m}}\right)$ is a 1-Eulerian tour of $\widetilde{G}$, then $\left(e_{1}, \ldots, e_{m}\right)$ is a $\pi$-Eulerian tour of $G$. Conversely, for each $\pi$-Eulerian tour of $G$, the occurrences of each edge $f$ in the tour can be labeled with an arbitrary permutation of $\left\{1, \ldots, \pi_{\operatorname{tail}(f)}\right\}$ to obtain a 1 -Eulerian tour of $\widetilde{G}$. Hence for a fixed edge $e$ with $\operatorname{tail}(e)=w$,

$$
\epsilon_{\pi}(G, e) \prod_{v \in V}\left(\pi_{v} !\right)^{d_{v}}=\epsilon_{\mathbf{1}}\left(\widetilde{G}, e^{1}\right) \pi_{w}
$$

The factor of $\pi_{w}$ arises here from the label of the starting edge $e$, and the observation that $\epsilon_{\mathbf{1}}\left(\widetilde{G}, e^{i}\right)$ does not depend on $i$. In particular, $G$ has a $\pi$-Eulerian tour if and only if $\widetilde{G}$ is Eulerian.

To complete the counting in the case when $\widetilde{G}$ is Eulerian, the BEST theorem gives the number of 1-Eulerian tours of $\widetilde{G}$ starting with $e^{1}$, namely

$$
\epsilon_{\mathbf{1}}\left(\widetilde{G}, e^{1}\right)=\widetilde{\kappa}_{w} \prod_{v \in V}\left(d_{v} \pi_{v}-1\right) !
$$

where

$$
\widetilde{\kappa}_{w}=\kappa_{w} \prod_{v \neq w} \pi_{v}
$$

is the number of spanning trees of $\widetilde{G}$ oriented toward $w$, since each spanning tree of $G$ oriented toward $w$ gives rise to $\prod_{v \neq w} \pi_{v}$ spanning trees of $\widetilde{G}$.

We conclude that

$$
\epsilon_{\pi}(G, e)=\pi_{w} \widetilde{\kappa}_{w} \prod_{v \in V} \frac{\left(d_{v} \pi_{v}-1\right) !}{\left(\pi_{v} !\right)^{d_{v}}}
$$

which together with (1) completes the proof.

The watchful reader must now be wondering, is there a suitable vector $\pi$ with positive integer entries in the kernel of the Laplacian? The answer is yes. Following Björner and Lovász, we say that a vector $\mathbf{p} \in \mathbb{N}^{V}$ is a period vector for $G$ if $\mathbf{p} \neq \mathbf{0}$ and $\Delta \mathbf{p}=\mathbf{0}$. A period vector is primitive if the greatest common divisor of its entries is 1 . 
Lemma 3. [5, Prop. 4.1] A strongly connected multigraph $G$ has a unique primitive period vector $\pi_{G}$. All entries of $\pi_{G}$ are strictly positive, and all period vectors of $G$ are of the form $n \pi_{G}$ for $n=1,2, \ldots$ Moreover, if $G$ is Eulerian, then $\pi_{G}=\mathbf{1}$.

Recall $\kappa_{v}$ denotes the number of spanning trees of $G$ oriented toward $v$. Broder [3] and Aldous [1] observed that $\kappa=\left(\kappa_{v}\right)_{v \in V}$ is a period vector! This result is sometimes called the 'Markov chain tree theorem'.

Lemma $4([1,3]) . \Delta \kappa=0$.

Lemmas 3 and 4 imply that the vector $\pi=\frac{1}{M_{G}} \kappa$ is the unique primitive period vector of $G$, where

$$
M_{G}=\operatorname{gcd}\left\{\kappa_{v}: v \in V\right\}
$$

is the greatest common divisor of the oriented spanning tree counts. Our next result expresses the minimal length of a multi-Eulerian tour in terms of $M_{G}$ and the number

$$
U_{G}=\sum_{v \in V} \kappa_{v} d_{v}
$$

of unicycles in $G$ (that is, pairs $(t, e)$ where $t$ is an oriented spanning tree and $e$ is an outgoing edge from the root of $t)$.

Theorem 5. The minimal length of a multi-Eulerian tour in a strongly connected multigraph $G$ is $U_{G} / M_{G}$.

Proof. The length of a $\pi$-Eulerian tour is $\sum_{v \in V} \pi_{v} d_{v}$. By Theorem 2 along with Lemmas 3 and 4 , there exists a $\pi$-Eulerian tour if and only if $\pi$ is a positive integer multiple of the primitive period vector $\frac{1}{M_{G}} \kappa$. The result follows.

A special class of multi-Eulerian tours are the simple rotor walks $[9,13,7,8,11]$. In a simple rotor walk, the successive exits from each vertex repeatedly cycle through a given cyclic permutation of the outgoing edges from that vertex. If $G$ is Eulerian then a simple rotor walk on $G$ eventually settles into an Eulerian tour which it traces repeatedly. More generally, if $G$ is strongly connected then a simple rotor walk eventually settles into a $\pi$-Eulerian tour where $\pi$ is the primitive period vector of $G$.

Trung Van Pham introduced the quantity $M_{G}$ in [11] in order to count orbits of the rotor-router operation. In [6] we have called $M_{G}$ the Pham index of $G$ and studied the graphs with $M_{G}=1$, which we called coEulerian graphs. The significance of $M_{G}$ is not readily apparent from its definition, but we argue in [6] that $M_{G}$ measures 'Eulerianness'. Theorem 5 makes this explicit, in that the minimal length of a multi-Eulerian tour depends inversely on $M_{G}$.

A consequence of Theorem 2 is that the number of $\pi$-Eulerian tours beginning with edge $e$ does not depend on head $(e)$. This can also be proved directly by cycling the tour to relate the number of tours starting with edge $e$ to the total number of $\pi$-Eulerian tours:

$$
\epsilon_{\pi}(G, e)=\frac{\pi_{\text {tail }(e)} \sum_{f \in E} \epsilon_{\pi}(G, f)}{\sum_{v \in V} \pi_{v} d_{v}} .
$$


We thank an anonymous referee for pointing out that the proof method of Theorem 2 also gives an efficient count of certain more general tours.

Definition 2. Fix a vector $\lambda \in \mathbb{N}^{E}$ with all entries strictly positive. A $\lambda$-Eulerian tour is a closed path that uses each directed edge $e$ exactly $\lambda(e)$ times.

Theorem 6. Let $G=(V, E)$ be a strongly connected directed multigraph, and let $\lambda \in \mathbb{N}^{E}$. Then $G$ has a $\lambda$-Eulerian tour if and only if

$$
\sum_{\operatorname{tail}(e)=v} \lambda_{e}=\sum_{\operatorname{head}(e)=v} \lambda_{e} \quad \text { for all } v \in V .
$$

If $G$ has a $\lambda$-Eulerian tour, then the number of $\lambda$-Eulerian tours starting with a fixed edge e with tail $w$ is

$$
\operatorname{det} \widetilde{\Delta}_{w} \frac{\lambda_{e} \prod_{v \in V}\left(\widetilde{d}_{v}-1\right) !}{\prod_{f \in E}\left(\lambda_{f}\right) !}
$$

where $\widetilde{\Delta}_{w}$ is the submatrix omitting row and column $w$ of the Laplacian of the multigraph $\widetilde{G}$ obtained by replacing each edge e of $G$ from $u$ to $v$ by $\lambda_{e}$ edges $e^{1}, \ldots$, e $e^{\lambda_{e}}$ from $u$ to $v$; and $\widetilde{d}_{v}=\sum_{\text {tail }(e)=v} \lambda_{e}$ is the degree of $v$ in $\widetilde{G}$.

Proof. If $\left(e_{1}^{i_{1}}, \ldots, e_{\ell}^{i_{\ell}}\right)$ is a 1-Eulerian tour of $\widetilde{G}$, then $\left(e_{1}, \ldots, e_{\ell}\right)$ is a $\lambda$-Eulerian tour of $G$. Conversely, for each $\lambda$-Eulerian tour of $G$, the occurrences of each edge $f$ in the tour can be labeled with an arbitrary permutation of $\left\{1, \ldots, \lambda_{f}\right\}$ to obtain a 1-Eulerian tour of $\widetilde{G}$. Hence for a fixed edge $e$ with $\operatorname{tail}(e)=w$,

$$
\epsilon_{\lambda}(G, e) \prod_{f \in E}\left(\lambda_{f}\right) !=\epsilon_{\mathbf{1}}\left(\widetilde{G}, e^{1}\right) \lambda_{e}
$$

In particular, $G$ has a $\lambda$-Eulerian tour if and only if $\widetilde{G}$ is Eulerian, which happens if and only if (2) holds.

To complete the counting in the case when $\widetilde{G}$ is Eulerian, the BEST theorem gives the number of 1-Eulerian tours of $\widetilde{G}$ starting with $e^{1}$, namely

$$
\epsilon_{\mathbf{1}}\left(\widetilde{G}, e^{1}\right)=\operatorname{det} \widetilde{\Delta}_{w} \prod_{v \in V}\left(\widetilde{d}_{v}-1\right) !
$$

where $\operatorname{det} \widetilde{\Delta}_{w}$ is the number of spanning trees of $\widetilde{G}$ oriented toward $w$ by the matrix-tree theorem.

So far we have assumed that $G$ is strongly connected. For our last result we drop this assumption, and count $\pi$-Eulerian paths which are permitted to start and end at different vertices.

Definition 3. Fix $\pi \in \mathbb{N}^{V}$ with all entries strictly positive, and vertices $a, z \in V$. A $\pi$-Eulerian path from $a$ to $z$ is a path $a=e_{1}, \ldots, e_{m}=z$ in which each edge $e$ appears exactly $\pi_{\text {tail }(e)}$ times. 
Theorem 7. Let $G=(V, E)$ be a directed multigraph with Laplacian $\Delta$, let $\pi \in \mathbb{N}^{V}$ and fix vertices $a \neq z$. Then $G$ has a $\pi$-Eulerian path from a to $z$ if and only if $(V, E \cup(z, a))$ is strongly connected and

$$
\Delta \pi=1_{a}-1_{z} .
$$

If $G$ has a $\pi$-Eulerian path from a to $z$, then the number of such paths is

$$
\epsilon_{\pi}(G, a \rightarrow z)=\kappa_{z} \frac{\left(d_{z} \pi_{z}\right) !}{\left(\pi_{z}\right) ! d_{z}} \prod_{v \in V-\{z\}} \frac{\left(d_{v} \pi_{v}-1\right) !}{\left(\pi_{v} !\right)^{d_{v}-1}\left(\pi_{v}-1\right) !}
$$

Proof. Let $G^{\prime}$ be the multigraph obtained from $G$ by adding a new vertex $w$ with one edge $(z, w)$, one edge $(w, a)$ and $\pi_{z}-1$ edges $(w, z)$. Set $\pi_{w}=1$. Given a $\pi$-Eulerian tour of $G^{\prime}$, omitting all edges incident to $w$ yields a $\pi$-Eulerian path from $a$ to $z$ in $G$. Conversely, any $\pi$-Eulerian path from $a$ to $z$ in $G$ can be augmented to a $\pi$-Eulerian tour of $G^{\prime}$ beginning with the edge $(w, a)$ (and necessarily ending with edge $(z, w))$ by inserting $\pi_{z}-1$ detours from $z$ to $w$ and back. (Here we have used $a \neq z$; in the case $a=z$ we would need to set $\pi_{w}=\pi_{z}$.) This insertion can be performed in $\left(\begin{array}{c}d_{z} \pi_{z}+\pi_{z}-1 \\ \pi_{z}-1\end{array}\right)\left(\pi_{z}-1\right)$ ! possible ways. Hence

$$
\epsilon_{\pi}\left(G^{\prime},(w, a)\right)=\epsilon_{\pi}(G, a \rightarrow z)\left(\begin{array}{c}
d_{z} \pi_{z}+\pi_{z}-1 \\
\pi_{z}-1
\end{array}\right)\left(\pi_{z}-1\right) !
$$

In particular, $G$ has a $\pi$-Eulerian path from $a$ to $z$ if and only if $G^{\prime}$ has a $\pi$-Eulerian tour. By Theorem 2, this happens if and only if $G^{\prime}$ is strongly connected and $\Delta^{\prime} \pi=\mathbf{0}$, where $\Delta^{\prime}$ is the Laplacian of $G^{\prime}$; equivalently, $(V, E \cup(z, a))$ is strongly connected and $\Delta \pi=1_{a}-1_{z}$.

For the count, since the spanning trees of $G^{\prime}$ oriented toward $w$ are in bijection with the spanning trees of $G$ oriented toward $z$, we obtain from Theorem 2

$$
\epsilon_{\pi}\left(G^{\prime},(w, a)\right)=\kappa_{z} \prod_{v \in V \cup\{w\}} \frac{\left(d_{v}^{\prime} \pi_{v}-1\right) !}{\left(\pi_{v} !\right)^{d_{v}^{\prime}-1}\left(\pi_{v}-1\right) !}
$$

where $d_{v}^{\prime}$ is the outdegree of $v$ in $G^{\prime}$. For $v \notin\{w, z\}$ we have $d_{v}^{\prime}=d_{v}$. Since $d_{w}^{\prime}=\pi_{z}$ and $\pi_{w}=1$, the ratio on the right side is just $\left(\pi_{z}-1\right)$ ! when $v=w$. Since $d_{z}^{\prime}=d_{z}+1$, we end up with

$$
\epsilon_{\pi}(G, a \rightarrow z)=\kappa_{z}\left(\begin{array}{c}
d_{z} \pi_{z}+\pi_{z}-1 \\
\pi_{z}-1
\end{array}\right)^{-1} \frac{\left(d_{z} \pi_{z}+\pi_{z}-1\right) !}{\left(\pi_{z} !\right)^{d_{z}}\left(\pi_{z}-1\right) !} \prod_{v \in V-\{z\}} \frac{\left(d_{v} \pi_{v}-1\right) !}{\left(\pi_{v} !\right)^{d_{v}-1}\left(\pi_{v}-1\right) !}
$$

which simplifies to (3).

\section{References}

[1] David Aldous, The random walk construction of uniform spanning trees and uniform labelled trees. SIAM J. Disc. Math. 3 450-465, 1990. 
[2] Arash Asadi and Spencer Backman, Chip-firing and Riemann-Roch theory for directed graphs, Electronic Notes Discrete Math. 38:63-68, 2011. arXiv:1012.0287

[3] Andrei Broder, Generating random spanning trees. Foundations of Computer Science, 30th Annual Symposium on, pages 442-447. IEEE, 1989.

[4] T. van Aardenne-Ehrenfest and N. G. de Bruijn, Circuits and trees in oriented linear graphs, Simon Stevin 28, 203-217, 1951.

[5] Anders Björner and László Lovász, Chip-firing games on directed graphs, J. Algebraic Combin. Vol 1. 305-328, 1992.

[6] Matthew Farrell and Lionel Levine, CoEulerian graphs, Proc. Amer. Math. Soc., to appear, 2015. arXiv:1502.04690

[7] Alexander E. Holroyd, Lionel Levine, Karola Mészáros, Yuval Peres, James Propp and David B. Wilson, Chip-firing and rotor-routing on directed graphs, In and Out of Equilibrium 2, Progress in Probability, Vol. 60, 331-364, 2008. arXiv:0801.3306.

[8] Alexander E. Holroyd and James G. Propp, Rotor walks and Markov chains, in Algorithmic Probability and Combinatorics, American Mathematical Society, 2010. arXiv:0904.4507

[9] V. B. Priezzhev, Deepak Dhar, Abhishek Dhar and Supriya Krishnamurthy, Eulerian walkers as a model of self-organised criticality, Phys. Rev. Lett. 77:5079-5082, 1996. arXiv: cond-mat/9611019

[10] Richard P. Stanley, Enumerative Combinatorics, vol. 2, Cambridge University Press, 1999.

[11] Trung Van Pham, Orbits of rotor-router operation and stationary distribution of random walks on directed graphs, Adv. Applied Math. 70:45-53, 2015. arXiv:1403.5875

[12] W.T. Tutte and C.A.B. Smith, On unicursal paths in a network of degree 4. Amer. Math. Monthly: 233-237, 1941.

[13] Israel A. Wagner, Michael Lindenbaum and Alfred M. Bruckstein, Smell as a computational resource - a lesson we can learn from the ant, 4th Israeli Symposium on Theory of Computing and Systems, pages 219-230, 1996. 